\title{
Kinetic Theory of a Confined Quasi-Two-Dimensional Gas of Hard Spheres
}

\author{
J. Javier Brey *, Vicente Buzón, Maria Isabel García de Soria and Pablo Maynar \\ Física Teórica, Universidad de Sevilla, Apartado de Correos 1065, 41080 Sevilla, Spain; \\ vbuzon@us.es (V.B.); gsoria@us.es (M.I.G.d.S.); maynar@us.es (P.M.) \\ * Correspondence: brey@us.es; Tel.: +34-954-55-0936
}

Academic Editors: Giancarlo Franzese, Ivan Latella, Miguel Rubi, Antonio M. Scarfone and Kevin H. Knuth Received: 25 October 2016; Accepted: 10 February 2017; Published: 14 February 2017

\begin{abstract}
The dynamics of a system of hard spheres enclosed between two parallel plates separated a distance smaller than two particle diameters is described at the level of kinetic theory. The interest focuses on the behavior of the quasi-two-dimensional fluid seen when looking at the system from above or below. In the first part, a collisional model for the effective two-dimensional dynamics is analyzed. Although it is able to describe quite well the homogeneous evolution observed in the experiments, it is shown that it fails to predict the existence of non-equilibrium phase transitions, and in particular, the bimodal regime exhibited by the real system. A critical revision analysis of the model is presented, and as a starting point to get a more accurate description, the Boltzmann equation for the quasi-two-dimensional gas has been derived. In the elastic case, the solutions of the equation verify an $\mathrm{H}$-theorem implying a monotonic tendency to a non-uniform steady state. As an example of application of the kinetic equation, here the evolution equations for the vertical and horizontal temperatures of the system are derived in the homogeneous approximation, and the results compared with molecular dynamics simulation results.
\end{abstract}

Keywords: kinetic theory; confined gases; $\mathrm{H}$ theorem

\section{Introduction}

In the last years, a particular geometry has attracted interest in the study of confined systems. It is a quasi-two-dimensional system of spherical particles placed between two large parallel plates separated by a distance $h$ smaller than two particle diameters. In the experiments, due to the inelasticity of collisions between macroscopic particles, a permanent injection of energy is needed to reach a steady state. This is done by vibrating the two parallel walls confining the system [1-4]. A scheme of the set-up is provided in Figure 1. When the dynamics of the projection of the grains' motion on the horizontal plane is considered, it looks like a two-dimensional fluid. Experiments show that the system obtained in this way remains homogeneous over a wide range of values of the parameters controlling the experiment. On the other hand, when increasing the average density and/or decreasing the intensity of the vibration, a series of non-equilibrium phase transitions show up [3-6]. Several models have been proposed in the literature trying to describe the rich observed phenomenology. The general idea is to formulate an effective two-dimensional dynamics that must include the mechanism of energy injection to compensate for the inelastic dissipation. An interesting phenomenological macroscopic approach has been presented in [7], focussed on explaining the existence of a bimodal regime characterized by a single dense cluster surrounded by a gas of quite agitated particles that is present in some experiments. The description incorporates both a temperature parameter in the horizontal plane and another temperature associated with the vertical motion. At a mesoscopic level, a stochastic approach has been introduced. The energy injection is described by means of an external noise term acting on the particles [8-10]. Although this model predicts the existence of a steady state, its relation with 
real experiments has not been established. Moreover, it does not conserve momentum. Notice that although the experiments are of course carried out with macroscopic inelastic particles, the theoretical analysis can always be particularized for molecular elastic fluids by considering the elastic limit. Although a model formulated for granular gases will be critically summarized and reviewed in the first part of the paper, in the second part, a Boltzmann kinetic equation for a quasi-two-dimensional gas composed of elastic hard spheres will be introduced. The main goal of the review of the granular model is to show that in order to describe what is observed in the experiments, one must go to a quite detailed description of the dynamics of the system. In addition, it also shows the way to proceed from the kinetic equation in order to get evolution equations describing the macroscopic dynamics of the system.

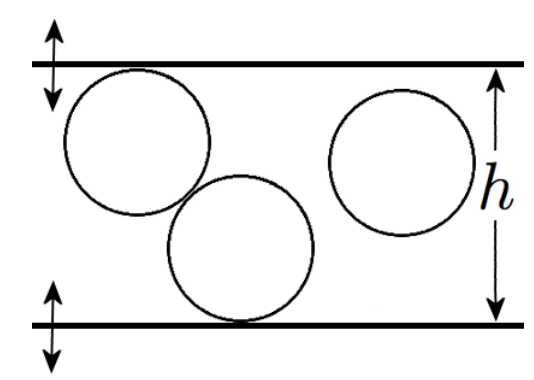

Figure 1. Sketch of the quasi-two-dimensional system described in the main text. The two parallel walls are vibrating, and the interest is on the dynamics observed when looking from above or below.

\section{A Collisional Model for the Effective Two-Dimensional Dynamics}

An approach to the real system closer to a mechanical description has been recently proposed [11,12]. The key point is to realize that the kinetic energy acquired by the particles by colliding with the vibrating walls is transferred to the horizontal degrees of freedom through the collisions between particles. Then, it is proposed to modify the usual two-dimensional collision rule for inelastic disks in order to incorporate a description of the energy transfer. The effective dynamics of the two-dimensional system is described by considering $N$ inelastic hard disks of diameter $\sigma$ moving on a surface $S$, such that when two of them with velocities $v_{1}$ and $v_{2}$ collide, their velocities are instantaneously changed to new values $v_{1}^{\prime}$ and $v_{2}^{\prime}$ given by

$$
\begin{aligned}
& v_{1} \rightarrow v_{1}^{\prime}=v_{1}-\frac{1+\alpha}{2} v_{12} \cdot \widehat{\sigma} \widehat{\sigma}+\Delta \widehat{\sigma}, \\
& v_{2} \rightarrow v_{2}^{\prime}=v_{2}+\frac{1+\alpha}{2} v_{12} \cdot \widehat{\sigma} \widehat{\sigma}-\Delta \widehat{\sigma} .
\end{aligned}
$$

Here $\alpha$ is the coefficient of normal restitution defined in the interval $0<\alpha \leq 1, v_{12} \equiv v_{1}-v_{2}$, and $\widehat{\sigma}$ is the unit vector in the direction of the centers of the two particles at contact. Finally, $\Delta$ is a positive characteristic speed. The presence of $\Delta$ is the only modification of the collision rule to account for kinetic energy transference from the vertical degree of freedom to the horizontal ones, so that by putting $\Delta=0$, the usual collision rule for smooth inelastic hard disks is recovered [13]. The variation of kinetic energy in a collision is

$$
\Delta e=m\left[\Delta^{2}-\alpha \Delta\left(\boldsymbol{v}_{12} \cdot \widehat{\sigma}\right)-\frac{1-\alpha^{2}}{4}\left(\boldsymbol{v}_{12} \cdot \widehat{\sigma}\right)^{2}\right],
$$

where $m$ is the mass of the particles. It is seen that energy can be gained or lost in a collision, depending on the relative velocity and the collision angle. The methods of non-equilibrium statistical mechanics developed for inelastic hard spheres and disks have been extended to the above collision rule [14]. In the low density limit, the dynamics of the system can be described by an inelastic Boltzmann 
kinetic equation. From it, an evolution equation for the granular temperature $T_{H}(t)$ of a homogeneous system — defined through the average kinetic energy [15]—can be derived. The granular temperature does not have the properties of the usual thermodynamical temperature, but still it is a useful quantity to describe the macroscopic state of the granular system. The evolution equation has the form

$$
\frac{\partial T_{H}(t)}{\partial t}=-\zeta_{H}(t) T_{H}(t)
$$

The rate of change of that granular temperature has the expression

$$
\zeta_{H}(t)=\sigma n_{H} v_{0 H}(t)(2 \pi)^{1 / 2}\left[\frac{1-\alpha^{2}}{2}-\alpha\left(\frac{\pi}{2}\right)^{1 / 2} \frac{\Delta}{v_{0 H}(t)}-\left(\frac{\Delta}{v_{0 H}(t)}\right)^{2}\right],
$$

with $n_{H}=N / S$ being the number density of disks and $v_{0 H}(t) \equiv\left(2 T_{H} / m\right)^{1 / 2}$ a thermal velocity. In order to derive this expression, the velocity distribution of the gas has been approximated by a Gaussian, which is quite accurate for not-very-inelastic particles. An expression incorporating some effects of the non-Gaussianity of the velocity distribution can be found in [14], and in a more compact form in the Appendix of Reference [16]. Equation (4) predicts the existence of a homogeneous steady state with a temperature

$$
T_{s t} \approx \frac{\pi m \alpha^{2}}{4\left(1-\alpha^{2}\right)^{2}}\left[1+\sqrt{1+\frac{4\left(1-\alpha^{2}\right)}{\pi \alpha^{2}}}\right]^{2} \Delta^{2} .
$$

Molecular dynamics (MD) simulations of the original three-dimensional system have been performed, and the state of the projected two-dimensional gas considered. By fitting the measured steady temperature to the above expression, the value of $\Delta$ in the model description has been determined [17]. Then, the obtained value is used into Equation (3) to compare with the simulation data for the relaxation of the temperature towards its steady value. This provides a test of the accuracy of the collisional model to describe the macroscopic two-dimensional dynamics of the system, at least in homogeneous situations. The results are shown in Figure 2. One of the plots corresponds to a system that initially is at a temperature larger than the stationary value, while in the other plot, an initial temperature below the stationary one is used. A quite good agreement between theory and simulation is found over all the relaxation of the temperature.

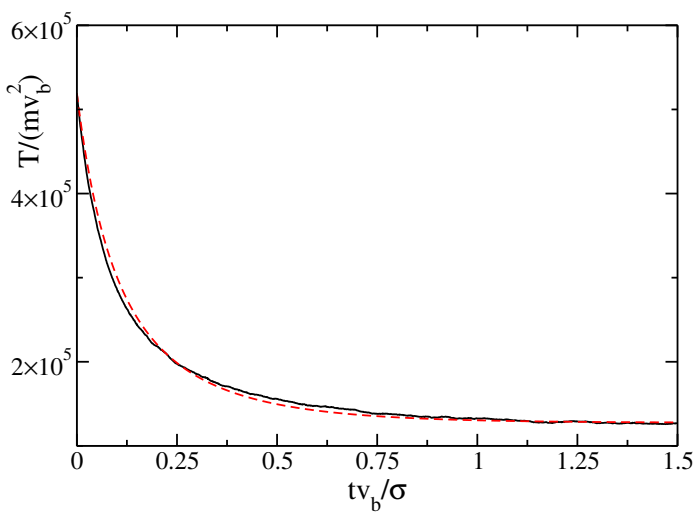

(a)

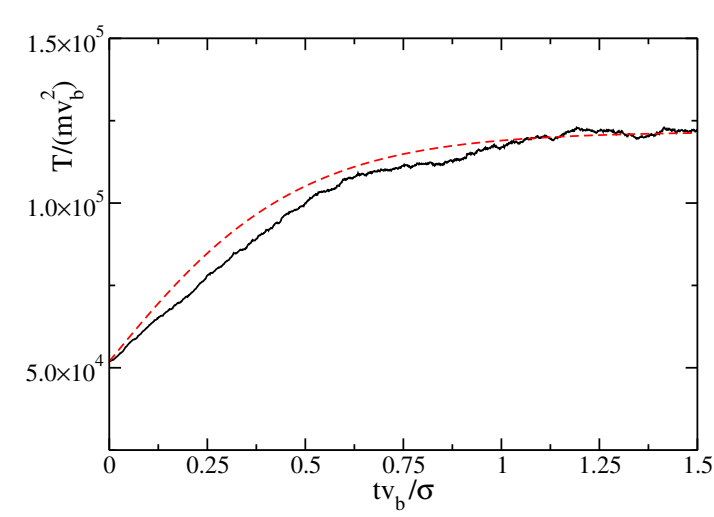

(b)

Figure 2. Relaxation of the granular temperature of the quasi-two-dimensional gas in a system with $\alpha=0.8, h=1.5 \sigma$, and three-dimensional density $n=0.02 \sigma^{-3}$. The walls are vibrating in a sawtooth way with a velocity $v_{b}$. Solid lines are the results from molecular dynamics (MD) simulations, while the dashed lines are the theoretical predictions obtained as indicated in the main text. (a) On the left hand side, a system cooling towards its steady granular temperature; (b) On the right hand side, the system started from a granular temperature smaller than the stationary one. 


\section{Hydrodynamic Equations}

To formulate a macroscopic theory of transport, an extension of the Chapman-Enskog method has been applied to the Boltzmann equation, to derive Navier-Stokes hydrodynamic equations in the low density limit. The ideas of the method are the same as for ordinary granular gases [18]. The obtained hydrodynamic equations for the number density $n(\boldsymbol{r}, t)$, velocity flow $\boldsymbol{u}(\boldsymbol{r}, t)$, and granular temperature $T(\boldsymbol{r}, t)$ read [19]

$$
\begin{gathered}
\partial_{t} n+\nabla \cdot(n \boldsymbol{u})=0, \\
\partial_{t} \boldsymbol{u}+\boldsymbol{u} \cdot \boldsymbol{\nabla} u+(m n)^{-1} \boldsymbol{\nabla} \cdot \mathrm{P}=0, \\
\partial_{t} T+\boldsymbol{u} \cdot \boldsymbol{\nabla} T+n^{-1}\left(\nabla \cdot \boldsymbol{J}_{q}+\mathrm{P}: \boldsymbol{\nabla} \boldsymbol{u}\right)=-T \zeta,
\end{gathered}
$$

where $\mathrm{P}(\boldsymbol{r}, t)$ is the pressure tensor, $J_{q}(\boldsymbol{r}, t)$ is the heat flux, and $\zeta(\boldsymbol{r}, t)$ is the local rate of variation of the temperature. They have the form

$$
\begin{gathered}
\mathrm{P}=n T \mathbf{I}-\eta\left[\boldsymbol{\nabla} \boldsymbol{u}+(\boldsymbol{\nabla} \boldsymbol{u})^{+}-\boldsymbol{\nabla} \cdot \boldsymbol{u l}\right], \\
J_{q}=-\kappa \nabla T-\mu \boldsymbol{\nabla} n, \\
\zeta=\zeta^{(0)}+\zeta_{1} \boldsymbol{\nabla} \cdot \boldsymbol{u} .
\end{gathered}
$$

In the above expressions, $\eta$ is the shear viscosity, $\kappa$ is the (thermal) heat conductivity, $\mu$ is a new transport coefficient peculiar to granular gases called diffusive heat conductivity, and $\zeta_{1}$ is another peculiar transport coefficient giving a Euler contribution to the hydrodynamic equations. All these coefficients depend on the temperature, and also on the coefficient of normal restitution $\alpha$ and the characteristic speed $\Delta$. Their expressions can be found in [19], and in a more concise way in the Appendix of [16]. They are given by the normal solutions-in the kinetic theory sense-of first-order partial differential equations. In Figures 3 and 4, dimensionless forms of the transport coefficients are plotted as a function of the dimensionless speed parameter $\Delta^{*} \equiv \Delta / v_{0}(t), v_{0}(t) \equiv(2 \mathrm{~T} / \mathrm{m})^{1 / 2}$, for $\alpha=0.85$. The value of the transport coefficients in the homogeneous steady state are indicated. The reduced coefficients are defined as

$$
\bar{\eta} \equiv \frac{\eta}{\eta_{0}}, \quad \bar{\kappa} \equiv \frac{\kappa}{\kappa_{0}}, \quad \bar{\mu} \equiv \frac{n \bar{\mu}}{T \kappa_{0}},
$$

where

$$
\eta_{0}=\frac{1}{2} \pi^{-1 / 2}(m T)^{1 / 2} \sigma^{-1}
$$

and

$$
\kappa_{0}=2 \pi^{-1 / 2}\left(\frac{T}{m}\right)^{1 / 2} \sigma^{-1}
$$

are the shear viscosity and the (thermal) heat conductivity, respectively, of a molecular gas of inelastic hard disks described by the Boltzmann equation, with the Boltzmann constant set equal to unity. Notice the non-monotonic behavior of $\zeta_{1}$ contrary to the other three transport coefficients, which are monotonic functions of the characteristic speed. Moreover, the change in sign of $\bar{\mu}$, which indicates a change in the direction of the energy flux generated by a density gradient, although it can look rather surprising, does not violate any fundamental symmetry property of the system or of the dynamical laws. 


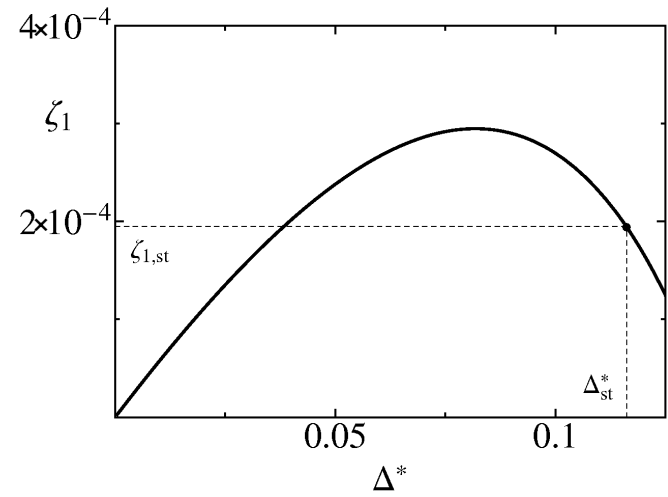

(a)

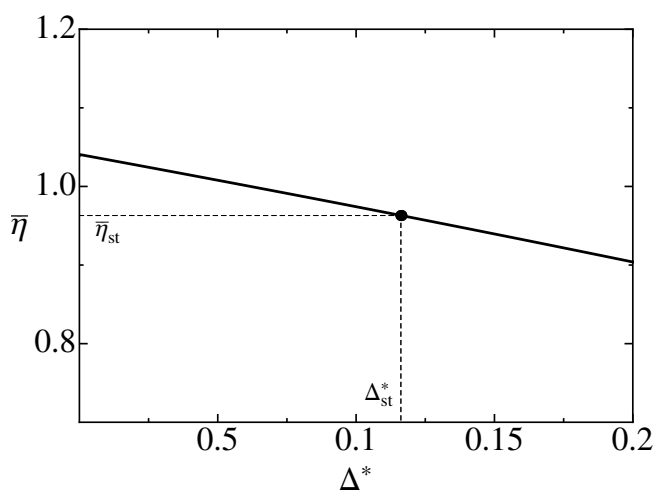

(b)

Figure 3. (a) Dimensionless Euler transport coefficient $\zeta_{1}$ as a function of the dimensionless characteristic speed $\Delta^{*}$ for the effective two-dimensional granular gas. The coefficient of normal restitution is $\alpha=0.85$. The dots indicate the value of the transport coefficients at the steady state, whose characteristic speed is $\Delta_{s t}^{*} ;(\mathbf{b})$ the same for the adimensionalized shear viscosity $\bar{\eta}$.

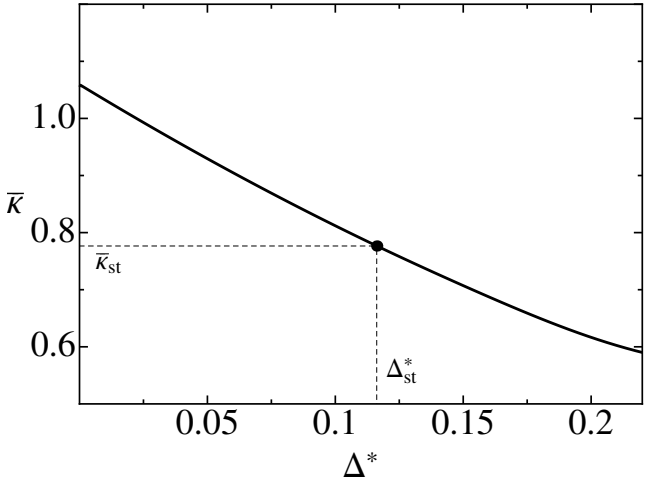

(a)

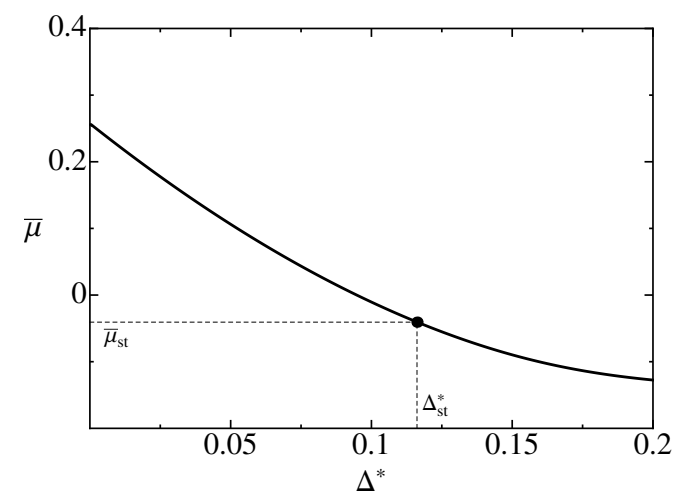

(b)

Figure 4. (a) Adimensionalized (thermal) heat conductivity $\bar{\kappa}$ as a function of the dimensionless characteristic speed $\Delta^{*}$ for the effective two-dimensional granular gas. The coefficient of normal restitution is $\alpha=0.85$. The dots indicate the value of the transport coefficients at the steady state, whose characteristic reduced speed is $\Delta_{s t}^{*} ;(\mathbf{b})$ the same for the adimensionalized diffusive heat conductivity $\bar{\mu}$.

\section{Stability Analysis}

One of the peculiarities of the model being considered is that-contrary to what happens with molecular fluids-it has a homogeneous hydrodynamics [20] defined by the macroscopic equations

$$
\partial_{t} n_{H}=0, \quad \partial_{t} \boldsymbol{u}_{H}=0, \quad \partial_{t} T_{H}=-\zeta_{H}^{(0)} T_{H}
$$

To investigate the stability of the hydrodynamic homogeneous states and see whether the model is able to predict any hydrodynamic instability, in agreement with what is observed in the experiments, the Navier-Stokes Equations (6)-(8) have been linearized around the homogeneous hydrodynamic trajectory. The coefficients of the resulting linear equations have a non-trivial time dependence through $\Delta^{*}$. This implies that both the eigenvalues and eigenfunctions of the dimensionless problem are time dependent, and the linear stability analysis is more involved than for smooth inelastic hard disks in the homogeneous cooling state [18]. An exception is the equation for the transversal component $\omega_{k \perp}$ of the scaled velocity field (shear mode), $\boldsymbol{\omega} \equiv \boldsymbol{u} / v_{0 H}(t)$, that is decoupled from the rest of equations and can be integrated directly, yielding [16] 


$$
\widetilde{\boldsymbol{\omega}}_{\boldsymbol{k} \perp}(s)=\widetilde{\boldsymbol{\omega}}_{\boldsymbol{k} \perp}(0) \exp \int_{0}^{s} d s^{\prime} \lambda_{\perp}\left(s^{\prime}\right),
$$

where

$$
\lambda_{\perp}(s) \equiv \frac{\bar{\zeta}^{(0)}}{2}-\bar{\eta}_{H} k^{2}
$$

In the above expression, a dimensionless time scale $s$ defined as

$$
d s=v_{0 H}(t) n_{H} \sigma d t
$$

is used. Moreover, the tilde indicates that a Fourier representation $k$ is being employed, as usual. From Equation (16) it follows that $\lambda_{\perp}<0$ for heating situations, in which the cooling rate is negative (the shear viscosity coefficient is always positive). Therefore, the scaled shear model is linearly estable for those processes in which the system heats towards the steady state. On the other hand, when the system is cooling $\lambda_{\perp}(s)$ is positive if $k<k_{\perp}(s)$, with

$$
k_{\perp}(s)=\left(\frac{\bar{\zeta}_{H}^{(0)}}{2 \bar{\eta}_{H}}\right)^{1 / 2} .
$$

The value of $k_{\perp}(s)$ changes in time. In the long time limit it goes to zero, since the cooling rate tends to zero while the shear viscosity remains finite and positive. The behavior of the other hydrodynamic fields cannot be analyzed analytically, but the linearized equations can be solved numerically. When the initial temperature of the system is smaller than its stationary value such that the temperature of the system increases monotonically in time, it is found [16] that the fields oscillate in time with a decaying amplitude (i.e., the system is linearly stable). On the other hand, when the temperature of the system is larger than the steady value, and the initial value of $k_{\perp}$ is larger than the wavenumber of the perturbation, the transversal component of the scaled velocity field initially grows in time, as predicted by Equation (16). Additionally, the amplitudes of the perturbations of the other field increase initially. Nevertheless, in the long time limit, all the perturbations decay to zero. This behavior is illustrated in Figure 5 for a system with $\alpha=0.8$ and an initial value of the scaled speed $\Delta_{0}^{*}=10^{-3}$. The initial value of $k_{\perp}$ is 0.91 , and the value of the wavenumber used is $k=0.2$.

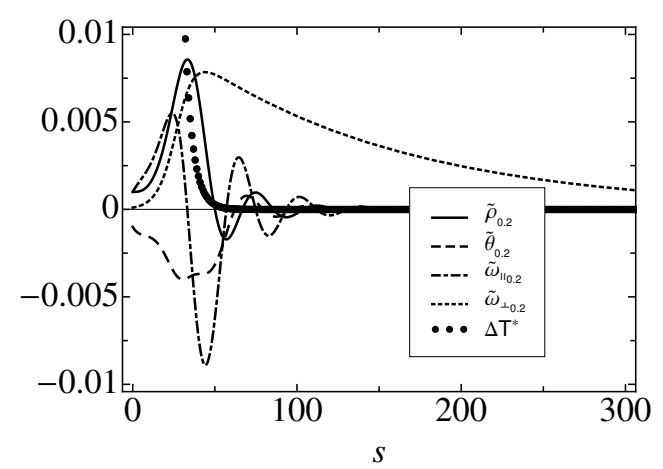

Figure 5. Time evolution of the dimensionless perturbations of the hydrodynamic fields, $\rho \equiv\left(n-n_{H}\right) / n_{h}$, $\boldsymbol{\omega} \equiv \boldsymbol{u} / v_{0}, \theta \equiv\left(T-T_{H}\right) / T_{H}$, as predicted by the linearized hydrodynamic equations. The tildes indicate Fourier transforms, and the time $s$ is a dimensionless scale defined from the original one by means of the thermal velocity $v_{0 H}$ and the mean free path, Equation (18). The wavenumber is $k=0.2$, while $k_{\perp}=0.91$. The initial temperature of the system is larger than its stationary value, so the system is monotonically cooling in time. The values of the initial perturbations of the fields are on the order of $10^{-3}$.

The point now is whether the amplitude of the perturbations grows enough during the initial period so as to render the linear approximation inaccurate, therefore being necessary to consider 
the complete nonlinear hydrodynamic equations. To address this point, we have carried out MD simulations of hard disks in a square of size $L$, using periodic boundary conditions. This imposes a minimum wave vector $k_{\text {min }}$. The spontaneous fluctuations occurring in the system were analyzed. The physical picture emerging from the analysis is [16] that the fluctuations of the traversal component of the velocity field with an amplitude that increases in time actually happen in the system. Their effect can manifest itself in the formation of clusters of particles. However, nonlinear couplings of hydrodynamic modes force the fluctuations to decay for large times, and the clusters to disappear if they were present. The conclusion is that all the homogenous hydrodynamic states of the collisional model-including the steady state-are stable, and consequently, it fails to explain the observed non-equilibrium phase transitions. In spite of this, we consider this model a relevant first step towards the understanding of the dynamics of confined granular gases starting from a description in terms of particles and using kinetic theory or non-equilibrium statistical mechanics, although some relevant ingredients are clearly missed in the model. In Figure 6, an example of the spatial configuration exhibited by the system as a consequence of a large spontaneous fluctuation of the transversal component of the velocity is shown. A large cluster of particles is clearly identified. It could be thought that it is the precursor of a bimodal regime, similar to the one observed in experiments. Nevertheless, the cluster disappears for larger times, and the system again becomes homogeneous in the stationary state. Notice that in order to explain the formation of the cluster and its posterior dilution, nonlinear hydrodynamic effects must be considered.

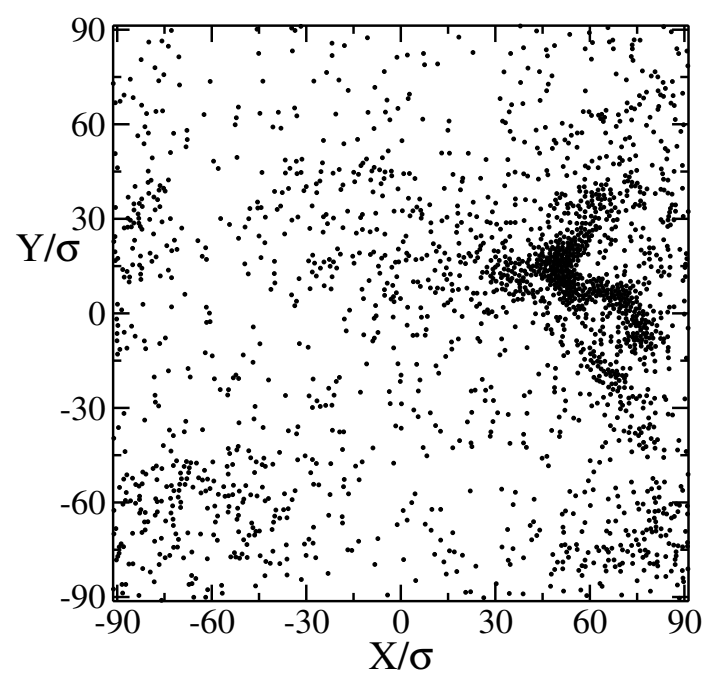

Figure 6. Snapshot of the positions of the particles in a system of $N=2000$ smooth inelastic hard disks. The parameters defining the system are $n=0.06 \sigma^{-2}, \alpha=0.6$, and $\Delta=2 \times 10^{-4} v_{0}(0) / \sqrt{2}$. At the time shown, the average accumulated number of collisions per particle was around 40.

\section{Back to the Origins: Boltzmann-Enskog Kinetic Equation}

The previous analysis shows that in order to progress, it is necessary to differentiate between confinement effects and inelasticity effects, aside from other possible relevant features. In a seminal paper, Schdmit and Löwen [21] derived several equilibrium properties of a system of hard spheres confined between two parallel plates separated by a distance between one and two particle diameters. The properties were particularly simple and exact in the low density limit. The natural question is whether we can formulate the dynamics of the system with the same degree of accuracy, at least in the low density limit. More precisely, is it possible to write down a "modified" Boltzmann equation for this special quasi-two-dimensional confinement? If this is the case, we have a solid starting point to investigate hydrodynamics and stability in confined systems, since there are several well-established procedures to derive hydrodynamics from a kinetic equation. 
By using a slight modification of the standard way of deriving the usual (bulk) Boltzmann and Enskog kinetic equations [22], it has been shown that the one particle distribution function $f(\boldsymbol{r}, \boldsymbol{v}, t)$ of the confined system of hard spheres in the low density limit satisfies the equation [23]

$$
\frac{\partial f}{\partial t}+v \cdot \frac{\partial f}{\partial r}=J[r, v \mid f]
$$

where the collision term $J$ is given by

$$
\begin{aligned}
J[\boldsymbol{r}, \boldsymbol{v} \mid f] \equiv & \sigma \int d \boldsymbol{v}_{1} \int_{\sigma / 2}^{h-\sigma / 2} d z_{1} \int_{0}^{2 \pi} d \varphi|\boldsymbol{g} \cdot \widehat{\boldsymbol{\sigma}}|\left[\theta(\boldsymbol{g} \cdot \widehat{\boldsymbol{\sigma}}) f\left(x, y, z_{1}, \boldsymbol{v}_{1}^{\prime}, t\right) f\left(\boldsymbol{r}, \boldsymbol{v}^{\prime}, t\right)\right. \\
& \left.-\theta(-\boldsymbol{g} \cdot \widehat{\boldsymbol{\sigma}}) f\left(x, y, z_{1}, \boldsymbol{v}_{1}, t\right) f(\boldsymbol{r}, \boldsymbol{v}, t)\right] .
\end{aligned}
$$

Here, $g \equiv v_{1}-v$, and $\theta$ is the Heaviside step function. Upon deriving the above equation, it has been assumed that no external force is acting on the system. Moreover, the walls confining the system are considered to be hard and at fixed positions. In this case, their effect can be described by means of boundary conditions to the kinetic equation [24]. Let us mention that, in principle, Equation (20) can be generalized for an arbitrary separation of the two parallel plates, although the mathematical description becomes much more involved. Define

$$
\begin{gathered}
H(t)=H^{(k)}(t)+H^{(c)}(t), \\
H^{(k)}(t)=\int d \boldsymbol{r} \int d \boldsymbol{v} f(\boldsymbol{r}, \boldsymbol{v}, t)[\ln f(\boldsymbol{r}, \boldsymbol{v}, t)-1], \\
H^{(c)}(t)=\frac{1}{2} \int d \boldsymbol{r} \int d \boldsymbol{r}_{1} n(\boldsymbol{r}, t) n\left(x, y, z_{1}, t\right) \theta\left(\sigma-\left|\boldsymbol{r}_{1}-\boldsymbol{r}\right|\right) .
\end{gathered}
$$

It is then proven [23] that in a system with elastic walls, $H(t)$ is bounded from below, and that $\partial_{t} H(t) \leq 0$, the equal sign holding only if

$$
f=f_{s t}(\boldsymbol{r}, \boldsymbol{v})=n(z) \varphi_{M B}(\boldsymbol{v}),
$$

where $\varphi_{M B}(v)$ is the Maxwellian velocity distribution,

$$
\varphi_{M B}(\boldsymbol{v})=\left(\frac{m}{2 \pi k_{B} T}\right)^{3 / 2} e^{-\frac{m v^{2}}{2 k_{B} T}} .
$$

The equilibrium density profile $n(z)$ is

$$
\left.n(z)=\frac{n_{0}(h-\sigma)}{b} \exp \left[a\left(z-\frac{h}{2}\right)\right)^{2}\right]
$$

with

$$
\begin{aligned}
n_{0} & =\frac{N}{A(h-\sigma)}, \\
a=\pi n_{0}(h-\sigma) \quad b & =\sqrt{\frac{\pi}{a}} \operatorname{erfi}\left[\sqrt{a}\left(\frac{h}{2}-\frac{\sigma}{2}\right)\right] .
\end{aligned}
$$

Here, erfi $(x)$ is the imaginary error function defined as

$$
\operatorname{erfi}(x) \equiv \pi^{-1 / 2} \int_{-x}^{x} d y e^{y^{2}}
$$

Equation (27) agrees with the low density limit of the expression given by Schmidt and Löwen [21]. Its accuracy has been checked by comparing it with MD simulation results. An example is provided in 
Figure 7. A quite good agreement is observed, especially taking into account the thinness of the system. The fact that the function $H(t)$ decreases monotonically in time for any solution of the kinetic equation suggests to define a time-dependent entropy for arbitrary states of the confined quasi-two-dimensional gas as

$$
S(t)=-k_{B} H(t) .
$$

The steady value of $S$ as defined above agrees—aside from an additive constant—with the result obtained by means of equilibrium statistical mechanics (e.g., ensemble theory or density functional theory) [21]. This is a non-trivial extension to an inhomogeneous confined system of the $H$ theorem verified by the Boltzmann equation.

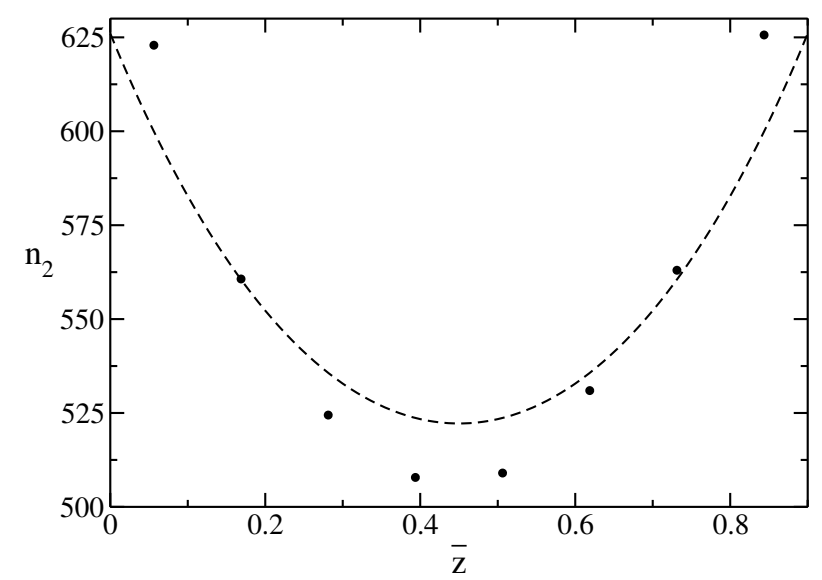

Figure 7. Density profile between the two plates. The symbols are simulation results, and the dashed line is the theoretical prediction given in the main text. The number of particles used in the simulations is $N=500$, the separation of the plates is $h=1.9 \sigma$, and the average density is given by $N \sigma^{2} / A=0.285$. The plotted dimensionless density is $n_{2}=n \sigma A$ and $\bar{z} \equiv(2 z-\sigma) / 2 \sigma$.

\section{Homogeneous Approximation: The Evolution of the Temperature}

It can be wondered how relevant the vertical inhomogeneity of the system is at a quantitative level. Here this will be investigated in the context of describing the relaxation of the temperature towards its equilibrium value. Then, we will restrict ourselves to the consideration of homogeneous (approximated) solutions of the kinetic Equation (20). Moreover, another simplification is introduced by assuming that the target particle is always located at $z=h / 2$. Thus, the equation being considered is

$$
\frac{\partial f(\boldsymbol{v}, t)}{\partial t}=J_{H}[v \mid f]
$$

with the homogeneous collision term being

$$
J_{H}[\boldsymbol{v} \mid f] \equiv \sigma \int d \boldsymbol{v}_{1} \int_{\sigma / 2}^{h-\sigma / 2} d z_{1} \int_{0}^{2 \pi} d \varphi|g \cdot \widehat{\boldsymbol{\sigma}}|\left[\theta(\boldsymbol{g} \cdot \widehat{\boldsymbol{\sigma}}) f\left(\boldsymbol{v}_{1}^{\prime}, t\right) f\left(\boldsymbol{v}^{\prime}, t\right)-\theta(-\boldsymbol{g} \cdot \widehat{\boldsymbol{\sigma}}) f\left(\boldsymbol{v}_{1}, t\right) f(\boldsymbol{v}, t)\right] .
$$

Notice that the effect of the confinement is still kept through the limitation in the angle between $g$ and $\widehat{\sigma}$, which is a function of the variable $z_{1}$, fixing the height of the incident particle at contact. The aim here is to derive evolution equations for the vertical and horizontal temperatures, $T_{z}$ and $T_{x y}$, respectively, defined as

$$
\begin{gathered}
n_{0} T_{z}(t) \equiv m \int d v v_{z}^{2} f(\boldsymbol{v}, t), \\
n_{0} T_{x y}(t) \equiv \frac{m}{2} \int d v\left(v_{x}^{2}+v_{y}^{2}\right) f(\boldsymbol{v}, t) .
\end{gathered}
$$


The (scalar) temperature of the system is $T=\left(T_{z}+2 T_{x y}\right) / 3$. Moreover, the distribution function will be approximated by a Maxwellian with two different temperature parameters; namely, $T_{x y}$ and $T_{z}$,

$$
f(\boldsymbol{v}, t) \approx \frac{n_{0}}{\pi^{3 / 2} \omega_{x y}^{2}(t) \omega_{z}(t)} e^{-\frac{v_{x}^{2}+v_{y}^{2}}{\omega_{x y}^{2}}-\frac{v_{z}^{2}}{\omega_{z}(t)^{2}}},
$$

where we have introduced the two thermal velocities

$$
\begin{gathered}
\omega_{x y}^{2}=\frac{T_{x y}(t)}{2 m}, \\
\omega_{z}^{2}=\frac{T_{z}(t)}{2 m} .
\end{gathered}
$$

Then, by taking the appropriate velocity moments in Equation (32), after some algebra it is obtained that

$$
\begin{aligned}
\frac{\partial T_{x y}}{\partial t} & =\frac{2 \sqrt{2 \pi} \beta^{3} m n_{0} \sigma^{2} \omega_{x y}}{3}\left(\omega_{z}^{2}-\omega_{x y}^{2}\right), \\
\frac{\partial T_{z}}{\partial t} & =\frac{4 \sqrt{2 \pi} \beta^{3} m n_{0} \sigma^{2} \omega_{x y}}{3}\left(\omega_{z}^{2}-\omega_{x y}^{2}\right) .
\end{aligned}
$$

In the above expressions,

$$
\beta \equiv \arcsin \frac{h-\sigma}{2 \sigma}
$$

is the maximum possible angle of $\widehat{\sigma}$ with the horizontal plane. To put the above results in a proper context, it is worth emphasizing that the starting kinetic equation does not admit homogeneous solutions, and consequently, the derived evolution equations for the two temperature parameters must be understood as first-order approximations. As expected, the equations show that the evolutions of the vertical and horizontal temperatures are coupled. Energy conservation requires that $T_{z}(t)+2 T_{x y}(t)$ be constant along the evolution of the system. Another point to underline is the nonlinear character of the equations.

To investigate the accuracy of Equations (39) and (40), their numerical solutions have been compared with MD simulations results. An example is provided in Figure 8 that corresponds to a system with density $n_{0}=0.06 \sigma^{-3}$ and initial temperatures $T_{z}(0)=3 T_{x y}(0)$. The separation of the two plates is $h=1.5 \sigma$. Notice that at all times it is $T_{z}(t)+2 T_{x y}(t)=3 T(0)$. It is observed that the theory reproduces the qualitative behaviour of the two temperature parameters well, although the predicted decay is definitely slower that the one shown by the simulation data, indicating that a more elaborated analysis of the kinetic equation is required to get a quantitative description of the evolution of the system. Of course, in the long time limit, both temperatures tend to the same value, as required by the discussion of the equilibrium state of the system in the previous section and the $H$ theorem.

The kinetic Equation (20) can be easily extended for inelastic hard spheres. The only modification to be done is the collision rule. Additionally, balance equations for the macroscopic fields density, velocity, and temperature can be derived. These equations involve formal expressions for the fluxes and the cooling rate. To close these equations leading to hydrodynamic equations, methods recently developed for granular gases $[25,26]$ seem easy to adopt for the present case. Once the Navier-Stokes equations are known, the stability issue can be addressed. Work along these lines is in progress. 


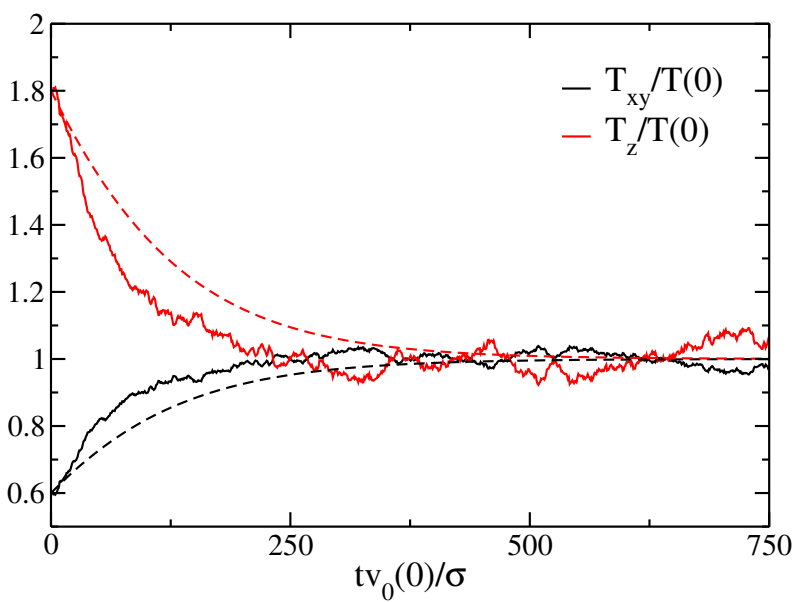

Figure 8. Decay of the vertical, $T_{z}$, and horizontal, $T_{x y}$, temperatures towards their equal steady values in a homogenous system. Time is measured in the dimensionless units indicated in the label, where $v_{0} \equiv \sqrt{2 T(0) / m}$. The solid lines are MD simulation results, while the dashed lines are the theoretical predictions given by Equations (39) and (40). Moreover, the two upper lines correspond to the vertical temperature, and the two lower lines to the horizontal temperature.

Acknowledgments: This research was supported by the Ministerio de Economía y Competitividad (Spain) through Gract No. FIS2014-53808-P (partially financed by FEDER funds).

Author Contributions: Vicente Buzón and Maria Isabel García de Soria carried out most of the numerical calculations and MD simulation, while J. Javier Brey and Pablo Maynar focused more on the development of the theory. In any case, all the autors were actively involved in the development of the whole project. All authors have read and approved the final manuscript.

Conflicts of Interest: The authors declare no conflict of interest.

\section{References}

1. Melby, P.; Reyes, V.F.; Prevost, A.; Robertson, R.; Kumar, P.; Egolf, D.A.; Urbach, J.S. The dynamics of thin vibrated granular layers. J. Phys. Condens. Matter 2005, 17, S2689-S2704.

2. Reis, P.M.; Ingale, R.A.; Shattuck, M.D. Crystallization of a Quasi-two-dimensional Granular Fluid. Phys. Rev. Lett. 2006, 96, 258001.

3. Rivas, N.; Ponce, S.; Gallet, B.; Risso, D.; Soto, R.; Cordero, P.; Mújica, N. Sudden Chain Energy Transfer Events in Vibrated Granular Media. Phys. Rev. Lett. 2011, 106, 088001.

4. Castillo, G.; Mújica, N.; Soto, R. Fluctuations and Criticality of a Granular Solid-Liquid-Like Phase Transition. Phys. Rev. Lett. 2012, 109, 095701.

5. Olafsen, J.S.; Urbach, J.S. Clustering, Order, and Collapse in a Driven Granular Monolayer. Phys. Rev. Lett. 1998, 81, 4369-4372.

6. Olafsen, J.S.; Urbach, J.S. Two-Dimensional Melting Far from Equilibrium in a Granular Monolayer. Phys. Rev. Lett. 2005, 95, 098002.

7. Khain, E.; Aranson, I.S. Hydrodynamics of a vibrated granular monolayer. Phys. Rev. E 2011, 84, 031308.

8. Willians, D.R.M.; MacKintoch, F.C. Driven granular media in one dimension: Correlations and equation of state. Phys. Rev. E 1996, 54, R9-R12.

9. Van Noije, T.P.C.; Ernst, M.H. Velocity distributions in homogeneous granular fluids: The free and the heated case. Granul. Matter 1998, 1, 57-64.

10. Puglisi, A.; Loreto, V.; Marini Bettolo Marconi, U.; Vulpiani, A. Kinetic approach to granular gases. Phys. Rev. E 1999, 59, 5582-5595.

11. Brito, R.; Risso, D.; Soto, R. Hydrodynamic modes in a confined granular fluid. Phys. Rev. E 2013, 87, 022209.

12. Soto, R.; Risso, D.; Brito, R. Shear viscosity of a model for confined granular media. Phys. Rev. E 2014, 90, 062204.

13. Goldhirsch, I. Rapid Granular Flows. Annu. Rev. Fluid Mech. 2003, 35, 267-293. 
14. Brey, J.J.; García de Soria, M.I.; Maynar, P.; Buzón, V. Homogeneous steady state of a confined granular gas. Phys. Rev. E 2013, 88, 062205.

15. Haff, P.K. Grain flow as a fluid-mechanical phenomenon. J. Fluid Mech. 1983, 134, 401-430.

16. Brey, J.J.; Buzón, V.; García de Soria, M.I.; Maynar, P. Stability analysis of the homogeneous hydrodynamics of a model for a confined granular gas. Phys. Rev. E 2016, 93, 062907.

17. Brey, J.J.; García de Soria, M.I.; Maynar, P.; Buzón, V. Memory effects in the relaxation of a confined granular gas. Phys. Rev. E 2014, 90, 032207.

18. Brey, J.J.; Dufty, J.W.; Kim, C.S.; Santos, A. Hydrodynamics for granular flow at low density. Phys. Rev. E 1998, 58, 4638-4653.

19. Brey, J.J.; Buzón, V.; García de Soria, M.I.; Maynar, P. Hydrodynamics for a model of a confined quasi-two-dimensional granular gas. Phys. Rev. E 2015, 91, 052201.

20. Brey, J.J.; Maynar, P.; García de Soria, M.I.; Buzón, V. Homogeneous hydrodynamics of a collisional model of confined granular gases. Phys. Rev. E 2014, 89, 052209.

21. Schmidt, M.; Lowën, H. Phase diagram of hard spheres confined between two parallel plates. Phys. Rev. E 1997, 55, 7228-7241.

22. Résibois, P.; de Leener, M. Classical Kinetic Theory of Fluids; John Wiley and Sons: New York, NY, USA, 1977.

23. Brey, J.J.; Maynar, P.; García de Soria, M.I. Kinetic equation and non-equilibrium entropy for a quasi-two-dimensional gas. Phys. Rev. E 2016, 94, 040103.

24. Dorfman, J.R.; van Beijeren, H. The Kinetic Theory of Gases. In Statistical Mechanics, Part B; Berne, B.J., Ed.; Plenum Press: New York, NY, USA, 1977; pp. 65-178.

25. Dufty, J.W.; Brey, J.J. Green-Kubo expressions for a granular gas. J. Stat. Phys. 2002, 109, 433-448.

26. Brey, J.J.; Ruiz-Montero, M.J. Simulation study of the Green-Kubo relations for dilute granular gases. Phys. Rev. E 2004, 70, 051301.

(C) 2017 by the authors; licensee MDPI, Basel, Switzerland. This article is an open access article distributed under the terms and conditions of the Creative Commons Attribution (CC BY) license (http:/ / creativecommons.org/licenses/by/4.0/). 